\title{
First Data from a Commercial Local Electrode Atom Probe
}

\author{
T. T. Gribb, J. D. Olson, R. L. Martens, J. D. Shepard, S. A. Wiener, T. C. Kunicki, R. M. Ulfig, \\ D. R. Lenz, E. M. Strennen, E. X. Oltman, J. H. Bunton, D. R. Strait, and T. F. Kelly
}

Imago Scientific Instruments Corporation, 6300 Enterprise Lane, Madison, WI 53719, http://www.imago.com/

The concept of a local electrode atom probe (LEAP) has its roots in the work of Nishikawa [1,2] in the early 1990's who invented the concept of a counter electrode proximal to a field evaporation specimen. Subsequent work by Kelly et al. [3,4] identified many advantages that accrue from operation of an atom probe with such a counter electrode. Various efforts in academic settings have been made by Nishikawa et al. [2], Kelly et al. [4], and Cerezo et al. [5] to demonstrate operational feasibility of such instruments. These experimental efforts have demonstrated that the key concepts of a LEAP work. However, there has heretofore not been a single instrument that integrates all of the potential functionality in a single instrument.

The Imago LEAP shown in Fig. 5 is an atom probe that is optimized to operate as a dedicated LEAP. It realizes the principle advantages of LEAP relative to conventional three-dimensional atom probes (3DAP), which are: 1) ease and rapidity of specimen preparation (at this time, analysis work is just beginning with microtip specimens) 2) high data collection rates (minutes), and 3) large solid angle of collection.

These advantages are illustrated in Fig. 1 through 4. Fig. 1 shows a 3D image (720,000 atoms) of the distribution of boron $(60 \mathrm{appm})$ in uniformly doped silicon. Fig. 2 shows a $2 \mathrm{D}$ hit map of silicon in which the 100 zone axis is visible. This 1 million atom hit map is part of 5 million atoms obtained from this high purity $(0.2 \Omega \mathrm{m})$ silicon. A $2 \mathrm{D}$ hit map of tungsten in which fine detail is evident in the 110 zone axis image is shown in Fig. 3a. The collection angle for Fig. 2 and $3 \mathrm{a}$ image is $\pm 25^{\circ}$ ( 0.59 steradian) which is more than an order of magnitude greater than energy compensated 3DAPs in operation today. Fig. 3b shows the 110 tungsten planes out to large solid angle in the side view of the $3 \mathrm{D}$ data set prior to correction for tip curvature. After correction, the planes become planar. Fig. 4 shows Ag impurities (100 appm) in Cu metal wire. This image contains 10 million atoms and is part of an 80 million atom 3D data set.

Each of these images was recorded at a data collection rate that exceeded 500 atoms per second. Fig. 4 was recorded at 10,000 atoms per second. A million atom dataset can thus be recorded in less than 5 minutes.

References

[1] O. Nishikawa et al., Appl. Surf. Sci. $76 / 77$ (1994) 424.

[2] O. Nishikawa, et al., Materials Charact.44 (2000) 29.

[3] T. F. Kelly et al., Ultramicroscopy 62 (1996) 29.

[4] T.F. Kelly et al., Materials Charact.44 (1999) 59.

[5] A. Cerezo et al., Rev. Sci. Instrum.71 (2000) 3016 


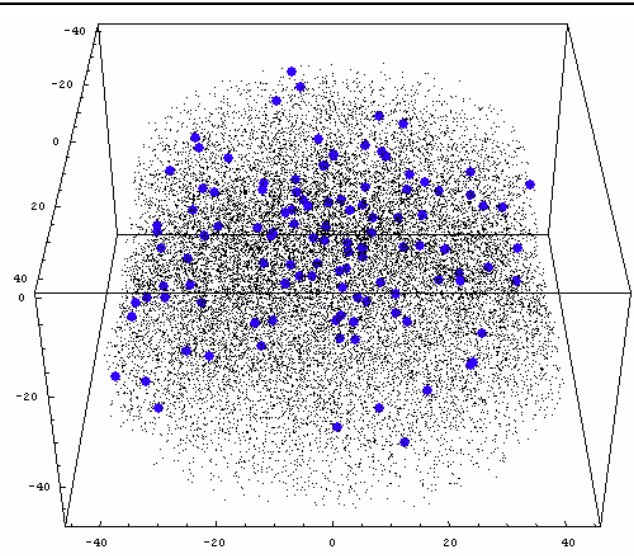

Fig. 1. 3D image of boron (blue dots) in uniformly doped silicon (black dots).

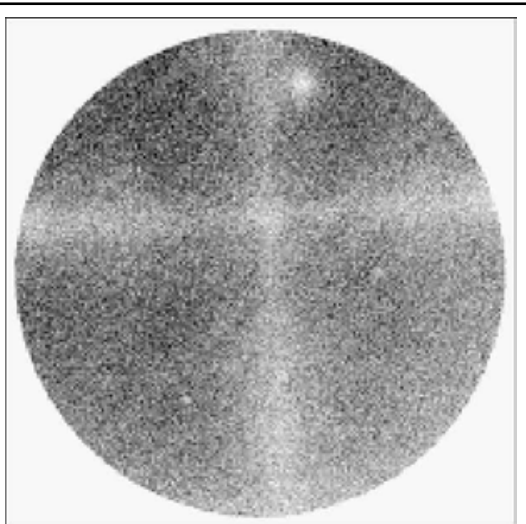

Fig. 2. 2D hit map of silicon atoms showing zone lines about the $\langle 100\rangle$ pole. This image is 1 million atoms of a 5 million-atom image.

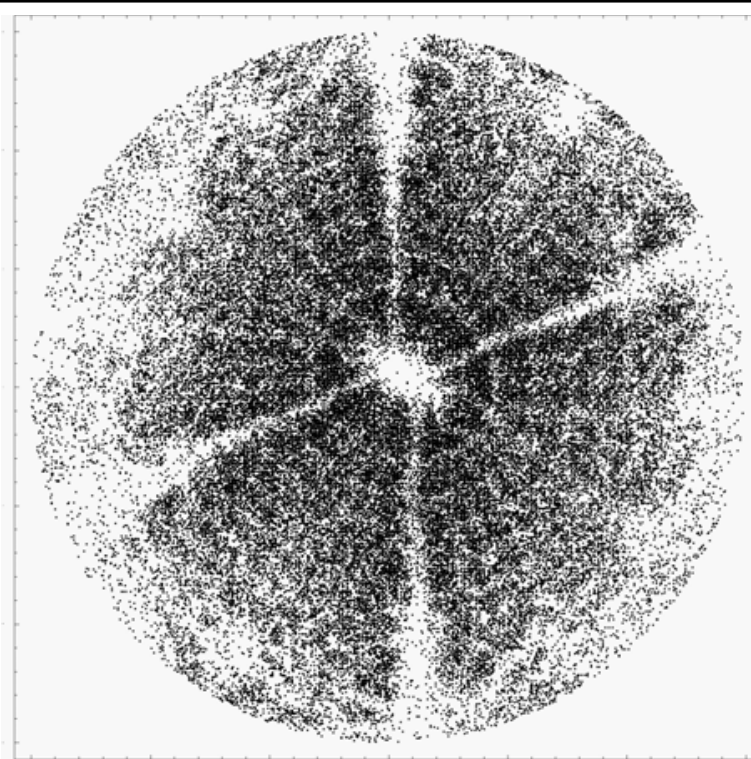

Fig. 3a. Top view 2D hit map from $<110>$ tungsten.

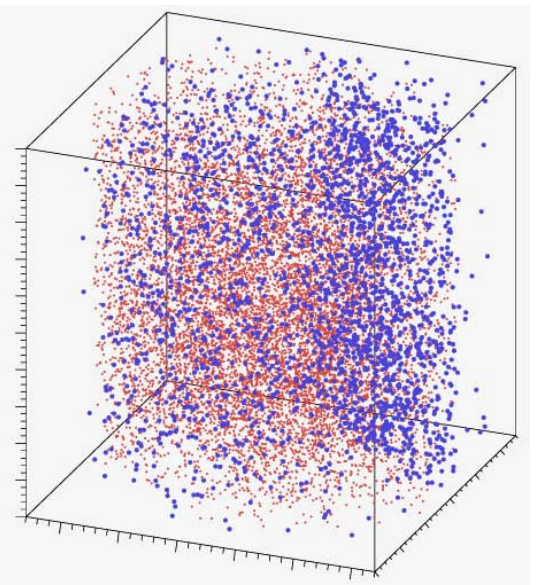

Fig. 4. Side view a 3D image from $\mathrm{Cu}$ wire showing $\mathrm{Ag}$ atoms and $0.1 \%$ of the $\mathrm{Cu}$ atoms. 10 million atoms in image.

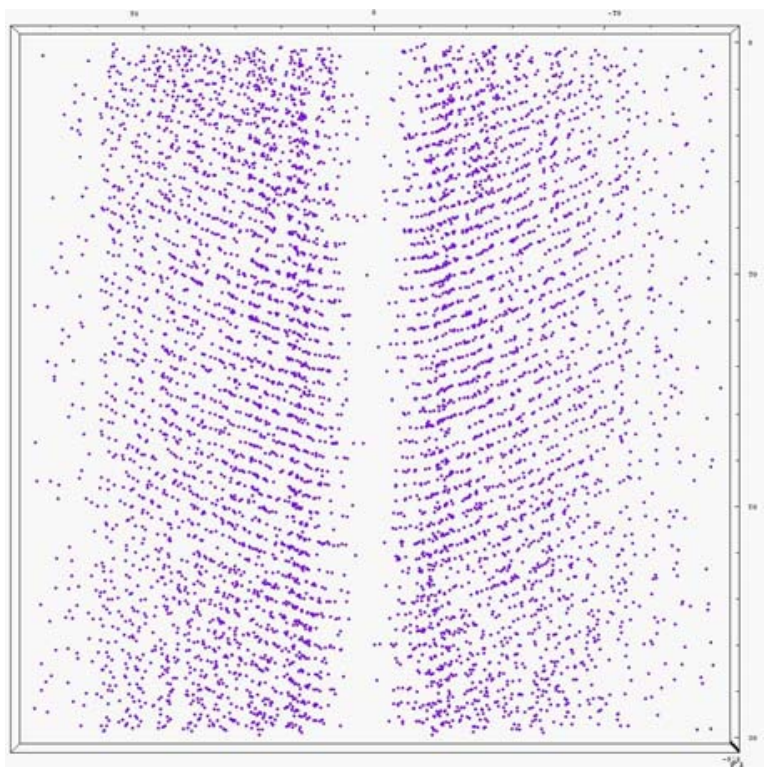

Fig. 3b. Side view section of 3D image showing 110 planes in tungsten.

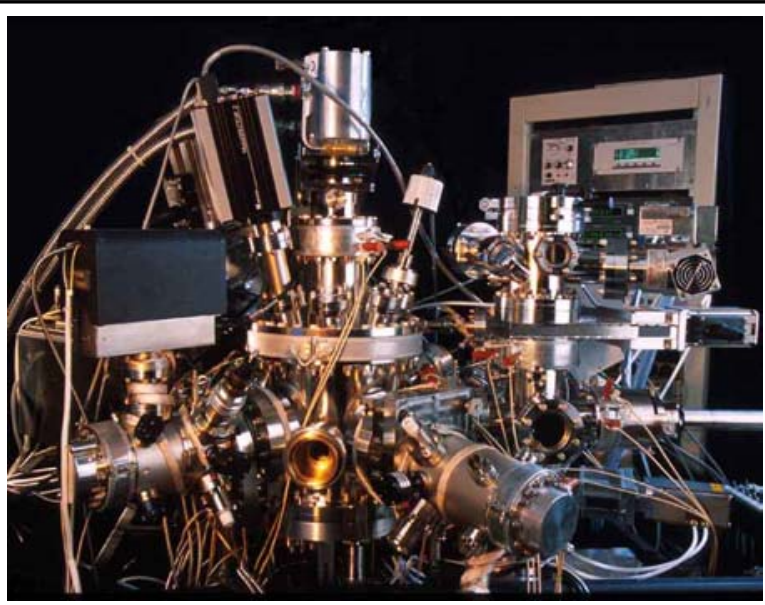

Fig. 5. Imago's Local Electrode Atom Probe 